\title{
Transcriptome analysis reveals distinct gene expression profiles in astrocytoma grades II-IV
}

\author{
Nato Narsia ${ }^{a}$, Pradeep Ramagirib ${ }^{\mathrm{b}}$ Jiri Ehrmann ${ }^{\mathrm{a}}$, Zdenek Kolar ${ }^{\mathrm{a}}$
}

\begin{abstract}
Background. Astrocytoma is the most prevalent form of primary brain cancer categorized into four histological grades by the World Health Organization. Investigation into individual grades of astrocytoma by previous studies has provided some insight into dysregulation of regulatory networks associated with increasing astrocytoma grades. However, further understanding of key mechanisms that distinguish different astrocytoma grades is required to facilitate targeted therapies.

Methods. In this study, we utilized a large cohort of publicly available RNA sequencing data from patients with diffuse astrocytoma (grade II), anaplastic astrocytoma (grade III), primary glioblastoma (grade IV), secondary glioblastoma (grade IV), recurrent glioblastoma (grade IV), and normal brain samples to identify genetic similarities and differences between these grades using bioinformatics applications.

Results. Our analysis revealed a distinct gene expression pattern between grade II astrocytoma and grade IV glioblastoma (GBM). We also identified genes that were exclusively expressed in each of the astrocytoma grades. Furthermore, we identified known and novel genes involved in key pathways in our study. Gene set enrichment analysis revealed a distinct expression pattern of transcriptional regulators in primary GBM. Further investigation into molecular processes showed that the genes involved in cell proliferation and invasion were shared across all subtypes of astrocytoma. Also, the number of genes involved in metastasis, regulation of cell proliferation, and apoptosis increased with tumor grade. Conclusions. We confirmed existing findings and shed light on some important genes and molecular processes that will improve our understanding of glioma biology.
\end{abstract}

Key words: astrocytoma grades II, III, IV, gene expression, RNA sequencing

Received: January 19, 2017; Accepted: April 18, 2017; Available online: April 26, 2017 https://doi.org/10.5507/bp.2017.020

${ }^{a}$ Department of Clinical and Molecular Pathology and Laboratory of Molecular Pathology, Faculty of Medicine and Dentistry, Palacky University Olomouc and University Hospital Olomouc, Czech Republic

bInstitute of Cancer and Genomic Sciences, College of Medical and Dental Sciences, University of Birmingham, Birmingham, United Kingdom Corresponding author: Pradeep Ramagiri,e-mail: pxr144@bham.ac.uk

\section{INTRODUCTION}

Astrocytoma is the most prevalent form of brain cancer $^{1}$. Previous studies have suggested that astrocytoma originates from cancer cells with stem-cell-like properties $^{2-3}$. The World Health Organization (WHO) classified astrocytoma into four histological grades (Grade I to IV) of increasing malignancy ${ }^{4}$. Grade II diffuse astrocytoma (AS II) is a slowly growing, invasive, semi-benign astrocytoma that is frequently diagnosed in younger patients, aged between 20 and 45 years and with an average age of 35 years $^{5}$. Histologically diffuse astrocytoma is not clearly distinguishable from surrounding normal tissue making it difficult for surgical resection ${ }^{6}$. Recurrence in most patients is observed after a few years, often progressed to more malignant grade III or grade IV Glioblastoma ${ }^{7-9}$. Grade III Anaplastic astrocytoma (AS III) is a fast growing, invasive, malignant astrocytoma. Tumor cells in AS III also vary in size and shape in comparison to AS II and were previously described to have an increased mitotic activity ${ }^{4}$. Mean age of patients diagnosed with AS III is 45 years. Patients undergo surgical resection, followed by chemotherapy and/or radiotherapy, as preferred means of treatment. Around $24 \%$ of the patients with AS III have an overall survival rate of five years ${ }^{10}$ with a median survival between one to four years ${ }^{11}$. The majority of AS III cases progress into grade IV glioblastoma after a few years ${ }^{7-8}$.

WHO grade IV Glioblastomas (GBM IV) are very fast growing and the most malignant type of astrocytoma. GBM IV can be characterized by the presence of necrosis and/or vascular proliferation. Glioblastoma IV can be genetically distinguished into two different types: 1). Primary GBM (pGBM IV), which occur rapidly without prior occurrences of lower grade astrocytoma $\mathrm{a}^{8,12}$ and constitutes the majority of the GBM IV. 2). Secondary GBM (sGBM IV), constitute only $5 \%$ of the total GBM IV cases and progress gradually from lower grade astrocytoma over several years ${ }^{13}$. Primary GBM is histologically indistinguishable from secondary GBM. However, sGBM are distinguishable from pGBM by the presence of IDH1 and IDH2 (Isocitrate Dehydrogenase 1/2 (NADP+)) gene mutations which are also present in AS II and AS III $\left(\right.$ ref. $\left.{ }^{14}\right)$. Poor survival rate is observed in patients with GBM with less than $5 \%$ of patients surviving longer than five years ${ }^{13}$.

There is an increased understanding of the pathobiology ${ }^{5,14-17}$, molecular subtypes ${ }^{18-20}$, and transcriptional regulatory networks ${ }^{21-25}$ of astrocytoma over the last two 
decades. Biomarkers that distinguish between astrocytoma grades have also been reported in previous studies ${ }^{26-27}$. Studies involving a comparative analysis of microarray data from AS II, AS III, and GBM IV provided insight into dysregulation of regulatory networks associated with increasing astrocytoma $\operatorname{grades}^{28}$.

However, the majority of these studies have either used no or a small number of normal brain samples in describing gene expression differences between astrocytoma grades ${ }^{20,26-28}$. In this study, with the help of publicly available RNA sequencing data from patients with AS II, AS III, and GBM IV, and normal brain samples, we aimed to describe genetic similarities and differences between these subtypes and also to identify key regulatory networks and signaling pathways that they share and also those that set them apart.

\section{MATERIAL AND METHODS}

\section{Samples}

RNA sequencing data used in this study was obtained from previously published studies as detailed in Table 1 . Normal samples included RNA sequencing data from 3 different studies that included, 2 epileptic patients, 5 Ambion Human Brain Reference RNA (HBRR), and 61 single cell (astrocytes) transcriptomes from epileptic patients. Astrocytoma grades II Diffuse astrocytoma, grade III anaplastic astrocytoma, and grade IV glioblastoma and corresponding normal brain samples were considered in this study (see supplementary Table 1 (S1)). No ethical approval was sought for this study as all the data used in this study was obtained from published studies that have obtained ethical approval.

\section{RNA sequencing data analysis}

Raw sequence reads were obtained from GEO (Gene Expression Omnibus; RRID: SCR_005012) in the form of FastQ files. Reads from FastQ files were aligned to human reference sequence hg19 (RRID: SCR_006553) using Rsubread aligner ${ }^{29}$. Mapped sequencing reads were assigned to hg19 refGene using featureCounts (RRID: SCR_012919). RefGene exon coordinates were obtained from UCSC table browser (RRID: SCR_013787). Differentially expressed genes were identified using edgeR (RRID: SCR_012802) $\left(\right.$ ref. ${ }^{30}$ ). Genes with read count per million $>1$ in at least half of the samples were considered for further analysis. The data was normalized using TMM (trimmed mean of $\mathrm{M}$ values) method.

\section{Gene set enrichment analysis}

Gene set enrichment analysis was done using Molecular Signatures Database (MSigDB; RRID: SCR_003199) to identify pathways and underlying biological themes. GSEA tool (RRID: SCR_003199) was used to investigate enrichment of 8 gene sets (tumor suppressor genes, oncogenes, translocated cancer genes, protein kinases, cell differentiation markers, homeodomain proteins, transcription factors/co-factors, and cytokine and growth factors) and the cancer census gene list ${ }^{31}$ in differentially expressed genes in our study.

\section{Pathway analysis}

Pathways enriched in differentially expressed genes were investigated using Molecular Signatures Database (MSigDB; RRID: SCR_003199). MSigDB evaluates the overlap of our genes sets with its collections and provides an estimate of the statistical significance ( $P$ and FDR q-value). We also used cytokine-cytokine interaction pathway (hsa (Homo sapiens): 04060) and glioma pathway (hsa: 05214) maps from Kyoto Encyclopedia of genes and genomes (KEGG; RRID: SCR_012773) to highlight key genes from our study.

\section{Statistical analysis}

Statistical analysis was done using the R language and environment (RRID: SCR_003005). The Heatmap3 program was used to generate the heat map for differentially expressed genes in R (ref. ${ }^{32}$ ).

\section{RESULTS}

\section{Similarities and differences in gene expression pattern in astrocytoma grades II-IV}

In our global gene expression analysis for grades II -IV astrocytoma, distinct gene expression pattern was observed between astrocytoma grade II and grade IV (Fig. 1). A total of 8936 over-expressed genes and 4699 under-expressed genes were observed in all AS II-IV subtypes when compared to all normal brain samples (log fold change $(\mathrm{FC}) \geq 2, P \leq 0.001$ and $\mathrm{FDR} \leq 0.002)$ (see

Table 1. List of samples used in this study.

\begin{tabular}{llcl}
\hline Sample (WHO grade) & Cited in this study as & Samples & GEO Accession no. \\
\hline Diffuse Astrocytoma (II) & A1-A34 & 34 & GSE48865 (Bao et al. 2014) \\
Anaplastic astrocytoma (III) & AA1-AA13 & 13 & \\
Primary glioblastoma (IV) & pGBM1-pGBM58 & 58 & \\
Secondary glioblastoma IV) & sGBM1-sGBM19 & 19 & \\
Recurrent glioblastoma (IV) & rGBM1-rGBM19 & 19 & \\
Single cell astrocytes & SC1-SC61 & 61 & GSE67835 (Darmanis et al. 2015) \\
Normal brain samples_1 & N1-N2 & 2 & GSE62731 (Stathias et al. 2014) \\
Normal brain samples_2 & N3-N7 & 5 & GSE49712 (Rapaport et al. 2013) \\
\hline
\end{tabular}


supplementary Table 2 (S2)). There was high overlap between grade IV subtypes in our study. Furthermore, we also observed a common expression pattern for grades II-IV samples for a group of genes (Fig. 1) (list of genes can be seen in supplementary Table 3 (S3)).

Differential gene expression analysis of astrocytoma grades AS II, AS III, pGBM, sGBM and rGBM revealed 773, 149, 3963, 280, and 304 over-expressed and 889, 200, 1859,377 , and 390 under-expressed genes, respectively $(\log \mathrm{FC} \geq 2, P \leq 0.001$, and FDR $\leq 0.002)$ (see supplementary Table 2 (S2)). The number of under-expressed genes was higher than the over-expressed genes in AS II, III, sGBM and rGBM, whereas in pGBM the number of overexpressed genes was more than that of under-expressed genes. The number of differentially expressed genes per sample in primary GBM was higher (3 fold for over-expressed genes) than the rest of the astrocytoma grades. 137 over-expressed genes and 191 under-expressed genes were common to all astrocytoma grades (Supplementary Fig. 1). The number of shared over-expressed and underexpressed genes in grade IV GBM was 247 and 330, respectively (Supplementary Fig. 2 and the list of genes can be seen in supplementary Table $3(\mathrm{~S} 3)$ ).
51 over-expressed genes and 42 under-expressed genes were found to be unique to AS II. Over-expressed genes include transcription factor genes (THRA, KLF15, and FHL1); protein kinase gene DCLK2; Cytokine and growth factor genes (IL17D, CMTM4 and SEMA6A); and Oncogenes (TFRC, MDM2, ETV5). Transcription factor gene ENO1 and cytokine and growth factor gene PTN were exclusively under-expressed in AS II. Overexpression of the ITM2C (BRI3) gene and under-expression of 3 genes; ADAM20, FAM133CP and LINC00504 were exclusive to AS III (see supplementary Table 3 (S3)). 3236 over-expressed and 1017 under-expressed genes were unique to grade IV glioblastoma subtypes. Genes previously reported in brain cancer, such as BIRC3, BRCA1, EGFR, ERBB2, PDGFR1B, and VEGFA were over-expressed and FGFR2 and NTKR3 were under-expressed exclusively in grade IV GBM, albeit, at varying degrees of significance (see supplementary Table $3(\mathrm{~S} 3)$ ). In primary GBM, 3192 and 994 genes were exclusively over-expressed and under-expressed, respectively (see supplementary Table 3 (S3)). SOX4 and SALL3 were only genes overexpressed in secondary GBM in our study. No underexpressed genes were found in our study. Out of 3 genes,

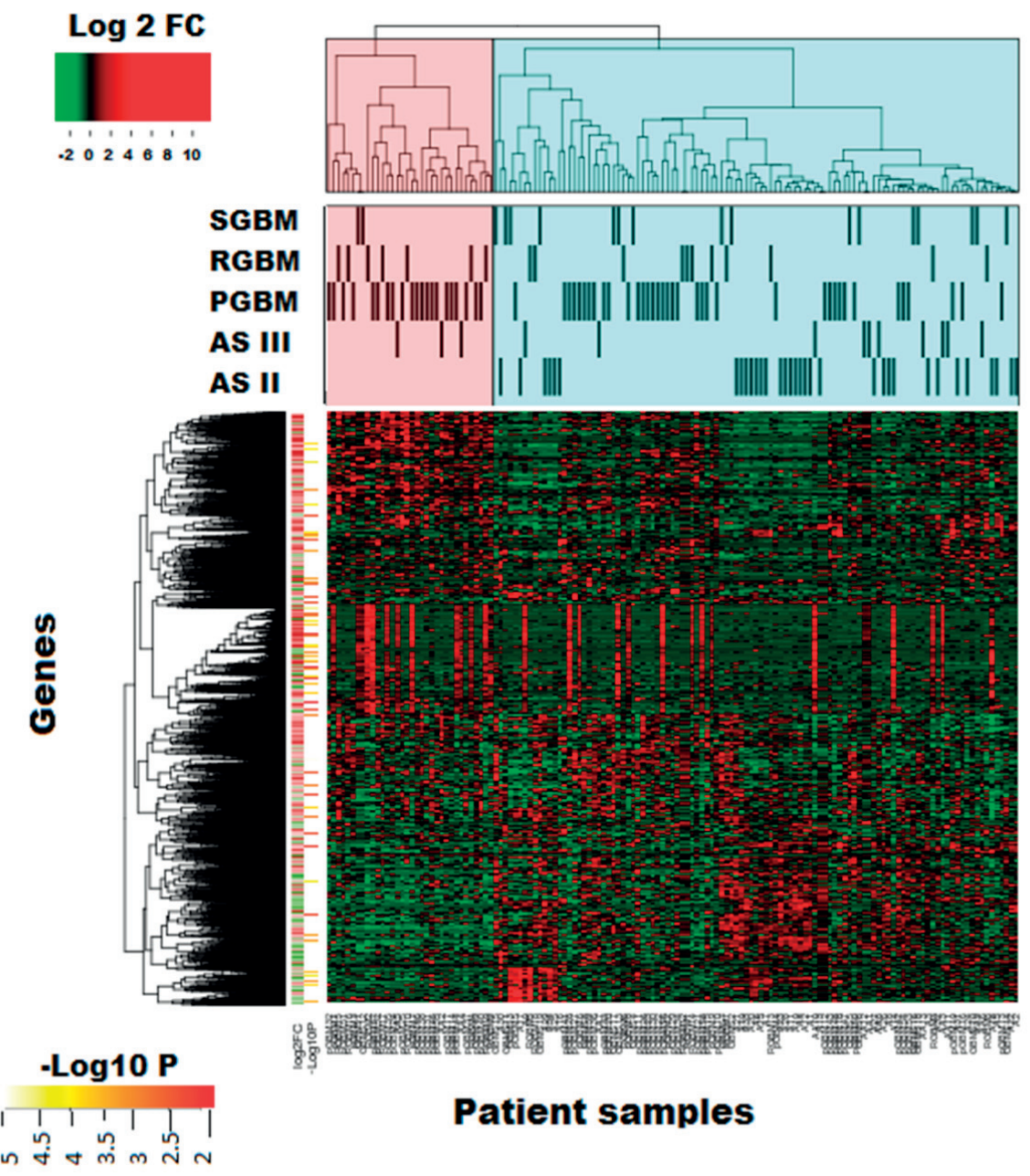

Fig. 1. Differential gene expression in AS II - IV. Heat map of differentially expressed genes in astrocytoma subtypes. Heat map of 13635 genes expressed across different subtypes showing a distinct gene expression pattern between grade II and grade IV astrocytoma samples. Shared gene expression between all grades can be seen as well. 
unique to recurrent GBM, FTL and CTSL genes were over-expressed and MT1JP gene was under-expressed.

\section{Gene expression pattern previously reported in glioma}

We found significant overlap $(P \leq 0.001)$ of differentially expressed genes in AS II-IV between our study and to those of a previously published study using microarray data $^{28}$ (see supplementary Table 4 (S4)). 36 genes were upregulated in grades II-IV astrocytoma in both studies. Among these 36 genes known cancer genes such as HIP1, HLA-A, MED12, and NOTCH1 genes were observed. AKT2 and EGFR genes were upregulated in grade IV astrocytoma in both studies. We observed 21 downregulated in grades II-IV astrocytoma in both studies which also include a cancer census gene WIF1. CALM2 (Calmodulin 2) gene was shared across all subtypes in both studies. Furthermore, we found 8 macrophage/microglia markers (TGFBI, CTSC, CD14, CD4, TLR2, ITGB2, TGFBR2, and $\mathrm{HEXB}$ ) upregulated in grade IV astrocytoma in our study (pGBM) and Seifert et al. study ${ }^{28}$. Macrophage markers were absent in shared genes for lower grade astrocytoma (AS-II and III) in these two studies (see supplementary Table 4 (S4)).

Furthermore, a literature review of the differentially expressed genes in our study revealed a similar expression pattern in genes (highlighted in bold letters in Table 2) that were previously implicated in glioma-genesis. Among these genes, MKI67 (Marker Of Proliferation Ki-67), EGFR (Epidermal Growth Factor Receptor), RTKs (Receptor tyrosine kinases), collagens, G-coupled receptor proteins genes and ligands, home box genes, chemokine receptors and ligands, and PDGF/VEGF growth factor family genes were among the most significantly over-expressed genes in AS II-IV (see Table 2 and supplementary Table 2 (S2)). EGFR gene is a well-known diagnostic marker for gliomas which is a receptor tyrosine kinase of the ErbB family, known to activate at least 4 major downstream signaling cascades including the RASRAF-MEK-ERK, PI3 kinase-AKT, PLC gamma-PKC and STATs modules. This gene was previously reported to be over-expressed in GBM and was significantly overexpressed in all GBM subtypes in our study (pGBM (4.06 $\log$ FC), sGBM (1.95 log FC), and rGBM (2.57 log FC). Also, SMARC4 (involved in controlling cell proliferation, migration, and invasion) which has been reported to be over-expressed in gliomas was significantly over-expressed in our study, (pGBM (1.87 log FC), sGBM (2.46 log FC), and rGBM (2.05 log FC) $\left(\right.$ ref. $\left.^{33}\right)$.

MALAT1 (Metastasis-associated lung adenocarcinoma transcript 1) was under-expressed in all astrocytoma grades in our study. It is a type of long noncoding RNA and its over-expression was reported to be associated with decreased metastasis and is a favorable prognostic factor as it plays a vital role in the reduction of extracellular

Table 2. List of 25 most significant differentially expressed genes in AS II-IV.

\begin{tabular}{|c|c|c|c|c|c|c|c|c|c|}
\hline \multicolumn{2}{|l|}{ AS II } & \multicolumn{2}{|c|}{ AS III } & \multicolumn{2}{|l|}{ PGBM } & \multicolumn{2}{|c|}{ SGBM } & \multicolumn{2}{|c|}{ RGBM } \\
\hline $\mathrm{Up}$ & Down & Up & Down & Up & Down & $\mathrm{Up}$ & Down & Up & Down \\
\hline OLIG1 & HIST1H4C & COL1A1 & GPC5-AS1 & COL3A1 & IDI2-AS1 & COL1A1 & GPC5-AS1 & COL1A1 & GPC5-AS1 \\
\hline FLJ16779 & LOC 101928590 & MIR6087 & LINC00499 & COL1A2 & GJB6 & MIR6087 & LINC00499 & MIR6087 & LINC00499 \\
\hline MIR6087 & IDI2-AS1 & SOD2 & LOC 101928590 & COL6A2 & WIF1 & RGMA & LOC 101928590 & FN1 & LOC 101928590 \\
\hline RGMA & CLDN10 & FKBP10 & WIF1 & IGFBP2 & GPR22 & FKBP10 & CLDN10 & FKBP10 & HIST1H4C \\
\hline SOX11 & MALAT1 & GFAP & HIST1H4C & FCGBP & CCDC144A & ELN & IDI2-AS1 & RGMA & CCDC144A \\
\hline HES6 & LOC 100289473 & HLA-B & GJB6 & TGFBI & GABRA2 & YBX1 & WIF1 & COL6A1 & IDI2-AS1 \\
\hline APOE & CCDC144A & COL6A1 & CCDC144A & $\mathrm{ABCC} 3$ & CLDN10 & ALYREF & CCDC144A & HLA-B & WIF1 \\
\hline LTBP3 & RYR3 & VEGFA & IDI2-AS1 & COL1A1 & ETNPPL & SEPN1 & ETNPPL & SOD2 & CLDN10 \\
\hline FAM181B & LRRC16A & APOE & SLC13A5 & MRC2 & MGAT4C & GFAP & SLC13A5 & ELN & GPR22 \\
\hline TSC22D4 & GABRA2 & FADS2 & CLDN10 & F2R & PWARSN & PFN1 & GJB6 & SEPN1 & GJB6 \\
\hline ADGRB1 & SAT1 & GPC1 & GABRA2 & MMP14 & GABRB1 & KHSRP & HIST1H4C & PFN1 & MGAT4C \\
\hline GFAP & SLC13A5 & TSC22D4 & GPR22 & MXRA5 & BMPR1B & HLA-B & SLCO1C1 & CHI3L1 & GABRA2 \\
\hline FADS2 & CPNE8 & TIMP2 & MALAT1 & CHI3L2 & MALAT1 & PRR11 & RYR3 & RPS3 & SLC13A5 \\
\hline A2M & LGI1 & MYO9B & ANKRD36C & PLAU & SLCO1C1 & EEF2 & MGAT4C & VEGFA & ETNPPL \\
\hline SLC12A4 & GJB6 & FN1 & LRRC16A & COL4A1 & RRN3P2 & TNRC18 & LRRC16A & SEC61A1 & GABRB1 \\
\hline SCRIB & EFNA5 & ТTYH3 & CACNB2 & COL5A1 & AQP4-AS1 & NOTCH1 & GABRA2 & HLA-H & PWARSN \\
\hline ZNF428 & GPR22 & SEC61A1 & PWARSN & CD163 & SLC13A5 & RAB5C & GPR22 & ALYREF & BMPR1B \\
\hline MTSS1L & LOC 101927692 & CHI3L1 & GABRB1 & MMP2 & ANKRD36C & LAMP1 & MALAT1 & GPC1 & LGI1 \\
\hline FLNA & $\mathrm{DIO} 2$ & YBX1 & ADAM20 & ANXA1 & LGI1 & SEC61A1 & LGI1 & YBX1 & SLCO1C1 \\
\hline GNB2 & FAS & HLA-H & MGAT4C & IFI30 & CACNB2 & RPSAP58 & BMPR1B & IGFBP7 & MALAT1 \\
\hline APC2 & LINC00632 & KCTD12 & SLC2A4 & NMB & EFNA5 & APOE & AQP4-AS1 & APOE & AQP4-AS1 \\
\hline PLEKHG2 & ANKRD36B & EEF2 & CPNE8 & SERPINE1 & LRRC16A & MYO9B & SLC $30 A 10$ & RAB5C & RYR3 \\
\hline SEPN1 & SLCO1C1 & ADGRB1 & SLC1A2 & IGFBP3 & SLC2A4 & TIMP2 & SLC2A4 & CTSD & LRRC16A \\
\hline EEF2 & GABRB1 & TNRC18 & LGI1 & PLVAP & ADGRV1 & ADGRB1 & EFNA5 & SURF4 & ANKRD36C \\
\hline TMEM259 & KLF4 & RPL27A & ANKRD36B & MDFI & ANKRD36 & PRKCSH & GABRB1 & ТTYH3 & CCDC144B \\
\hline
\end{tabular}

${ }^{*}$ Genes that were previously reported in astrocytoma were highlighted in bold letters.

** All Up-regulated genes in this table has $\log \mathrm{FC}$ of $>3.7, P \leq 0.000002$ and FDR $\leq 0.00002$ )

$* * *$ All downregulated genes in this table has $\log \mathrm{FC}$ of $<-5, P \leq 0.00002$ and FDR $\leq 0.000009$ ) 
signal-regulated kinase/mitogen-activated protein kinase (ERK/MAPK) signaling activity and expression of matrix metalloproteinase 2 (MMP2) $\left(\right.$ ref. $^{34}$ ). GJB6 (gap junction beta-6) gene was significantly under-expressed in all astrocytoma grades in our study and was previously reported to be deleted and/or mutated in glioma ${ }^{35}$. WIF1 (Wnt inhibitory factor 1) gene is a candidate tumor suppressor gene in glioblastoma and was reported to be downregulated in most glioblastomas ${ }^{36}$. WIF1 gene was under expressed in all AS grades in this study (Table 2). SLC1A2 (Solute Carrier Family 1 Member 2) gene was under-expressed in all astrocytoma grades in our study. Downregulated SLC1A2 gene assists in metastasis in glioma ${ }^{37}$. Underexpressed BMPR1B (bone morphogenetic protein receptor IB subunit) gene may reduce the malignancy of glioblastoma cells by upregulation of p21 and p27Kip 1 $\left(\right.$ ref. $\left.^{38}\right)$. BMPR1B was under-expressed in all astrocytoma grades in this study. LAMP1 (Lysosomal-associated membrane protein-1) gene was significantly over-expressed in all astrocytoma subtypes in our study (Table 2). This gene may play a role in metastasis in astrocytoma ${ }^{39}$. PFN1 (Phosphorylation of profilin-1) gene directs the angiocrine expression and glioblastoma progression through HIF- $1 \alpha$ accumulation ${ }^{40}$ and was over-expressed in grade IV glioblastoma subtypes in our study. YBX1 (Y-box binding protein-1) gene was over-expressed in all astrocytoma grades in our study (Table 2). It is a member of the cold shock protein family and functions in transcription and translation and is reported to be highly expressed in tumor cells and as a marker for tumor aggressiveness and clinical prognosis in various types of cancers, including glioblastoma ${ }^{41}$. CDH4 gene, which encodes for a cell adhesion protein involved in neural outgrowth and brain segmentation was under-expressed in AS II (log FC -1.97) $\left(\right.$ ref. $\left.^{42}\right)$. Notch ligand DLL3 known for its function in neurogenesis and glioma biology was over-expressed (log FC 8.99) (ref. $\left.{ }^{43}\right)$. Apoptotic gene GAS2 (log FC -2.41) and SHROOM2 (log FC -2.21), involved in cell spreading, were under-expressed. Gene NR2E1, involved in anterior brain differentiation, was under-expressed (log FC -1.57). Under-expression of NR2E1 gene was previously reported to be associated with cancer stem cell death and longer survival of G-CIMP glioma patients ${ }^{44}$.

\section{Gene set enrichment analysis of differentially expressed} genes in AS II-IV

Gene set enrichment analysis of 8 selected MSigDB gene family gene sets (as described in methods) and cancer census gene list revealed significant enrichment of all gene sets except tumor suppressor genes $(P \leq 0.001)$ for over-expressed genes in astrocytoma grades II-IV using. Significant depletion $(P \leq 0.001)$ of cell differentiation markers, homeodomain proteins, transcription factors/cofactors, and cytokine and growth factors was observed in the under-expressed genes (Fig. 2). Oncogenes and cancer census gene sets were significantly enriched $(P<0.05)$ in both over-expressed and under-expressed genes in each astrocytoma subtype (Supplementary Fig. 3 and 4). Tumor suppressor genes were not significantly enriched/depleted in any subtype except in over-expressed genes of pGBM $(P$

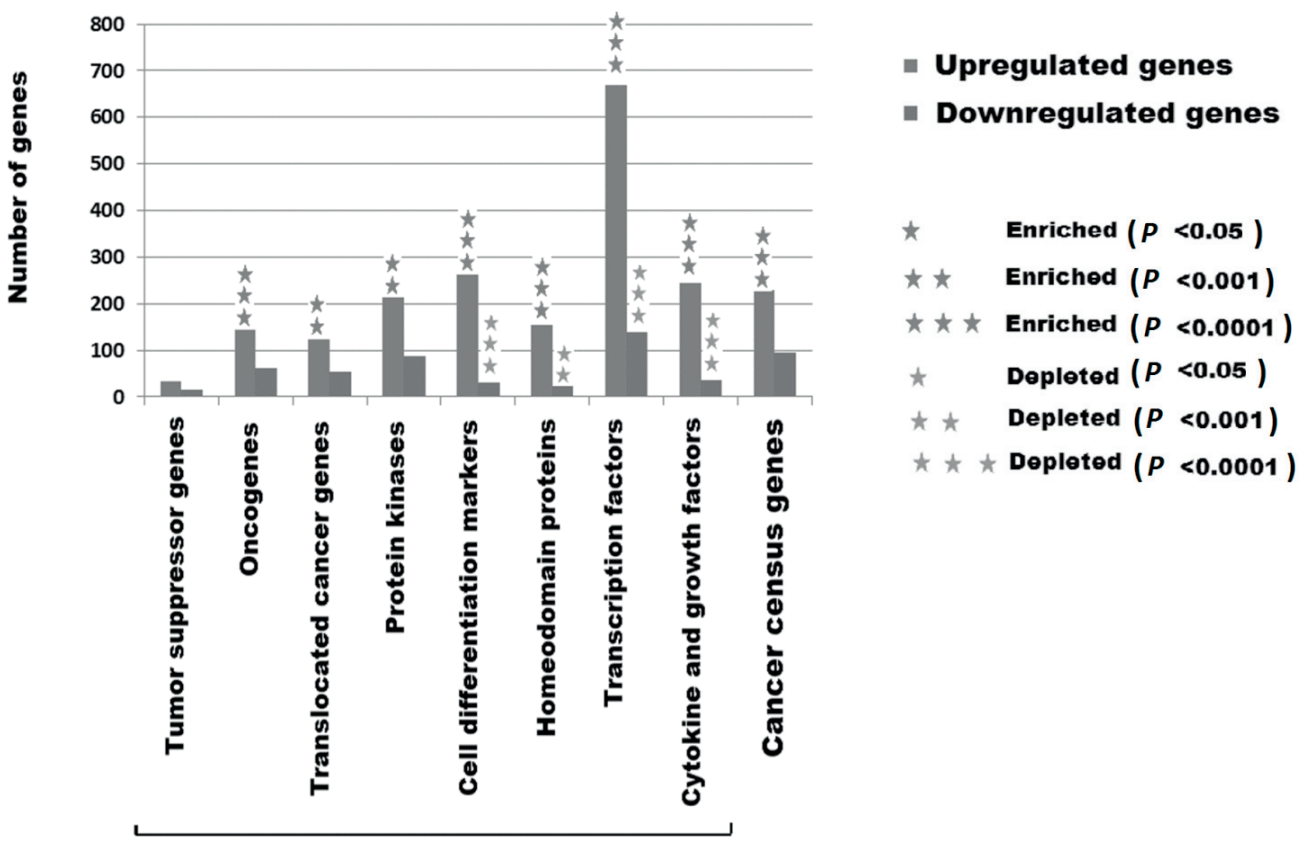

Functional category

Fig. 2. Functional annotations for all astrocytoma genes. Enrichment of tumor suppressor genes, oncogenes, translocated cancer genes, protein kinases, cell differentiation markers, homeodomain proteins, transcription factors and cytokine and growth factors; and Cancer Census Genes in differentially expressed genes in astrocytoma. Significant enrichment of genes in a category within a tumor type is represented by '*' $(P<0.05),{ }^{* * *},(P<0.01)$ and ${ }^{\prime * * *},(P<0.001)$ (Fisher's exact test). 


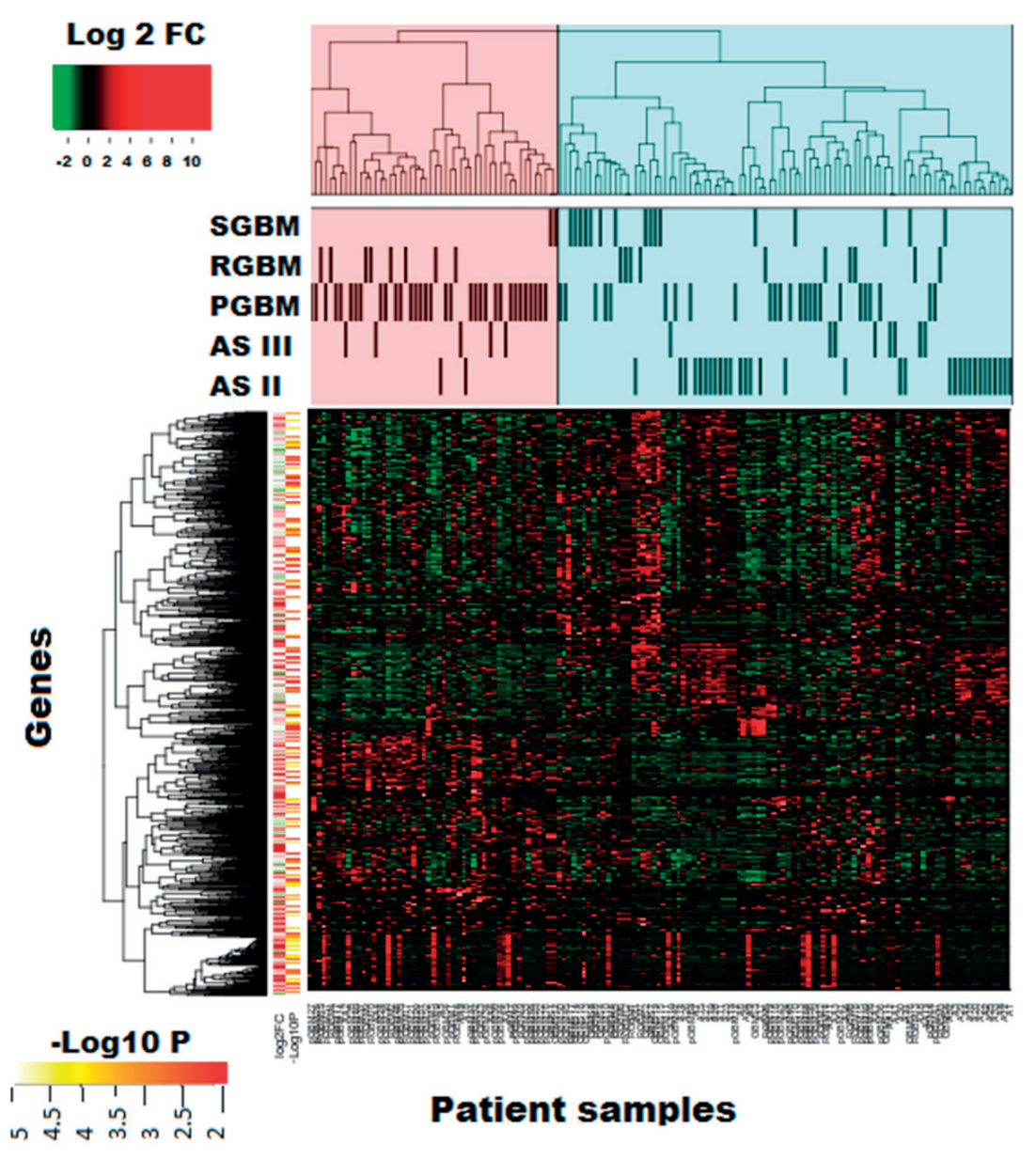

Fig. 3. Expression of transcription factors in AS II - IV. Heat map of transcription factors in different grades of astrocytoma. Heat map was generated from a list of 1446 transcription factor genes in different astrocytoma grades. A clear difference between grade II and grade IV was observed. Primary GBM samples showed a different expression pattern when compared to grade II astrocytoma. A cluster of transcription factor genes were shared among grade IV subtypes.

$=0.049)($ Supplementary Fig. 3). Homeodomain proteins were not significantly enriched/depleted in any subtype except in pGBM, where they were significantly depleted in over-expressed genes $(P \leq 0.001)$ (Supplementary Fig. 3). Significant enrichment of translocated cancer genes was observed in all AS subtypes except in the under-expressed genes of recurrent GBM. Transcription factors/cofactors were significantly enriched in all subtypes except in the over-expressed genes in AS III and under-expressed genes in sGBM). Significant depletion of transcription factors/cofactors was observed in over-expressed genes of pGBM (Supplementary Fig. 3 and 4). Protein kinases were significantly enriched in under-expressed genes in all GBM, however, enrichment was also observed in the overexpressed genes of pGBM and AS II. Enrichment of cell differentiation markers was seen in under-expressed genes of AS II and pGBM, as well as in the over-expressed genes of AS II and all GBM. Cytokine and growth factors were significantly enriched in under-expressed genes of pGBM only (Supplementary Fig. 4). Significant enrichment of tumor suppressor genes, oncogenes, protein kinases, and translocated cancer genes was observed in both overexpressed and under-expressed genes unique to pGBM. Significant enrichment (in over-expressed genes) and de- pletion (in under-expressed genes) of cell differentiation markers was observed in $\mathrm{pGBM}$ and vice versa for transcription factors/cofactors $(P<0.05)$. Significant depletion of homeodomain proteins (in over-expressed genes) and of cytokine and growth factors (in under-expressed genes) unique to pGBM was observed $(P<0.05)$.

\section{Expression of transcription factors in astrocytoma grades II-IV}

Transcription factors/co-factors was the most abundantly expressed group of genes in astrocytoma grades our study (Fig. 2). Distinct gene expression patterns were observed between AS II and all AS IV GBM for the 1446 transcription factor (TF) genes (Fig. 3). Primary GBM samples showed a different expression pattern compared to that of AS II and secondary GBM. A group of secondary GBM samples showed a similar gene expression pattern to that of AS II (Fig. 3). TFs in AS II were significantly enriched in cell cycle control, DNA repair, TGF BETA signaling, hypoxia, proliferation and apoptosis. Known oncogenes such as BRD4, CIC, ETV1, PATZ1, and PML and tumor suppressor gene KDM5C were upregulated, whereas oncogenes such as ETV5 and NFIB were downregulated in AS II. In AS III, TF genes 
were enriched in cell cycle (E2F) targets and chromatin binding. Oncogenes JUN (upregulated) and NFIB (downregulated) were observed in AS III subtype. Significant enrichment of TF genes involved in cell cycle control, DNA repair, TGF BETA signaling, hypoxia, proliferation, invasion, IL2-STAT5 pathway and apoptosis was seen in primary GBM. Secondary GBM showed enrichment of TF genes in pathways similar to those of primary GBM in our study. TF genes in recurrent GBM significantly enriched in cell cycle control, TGF BETA signaling, hypoxia, proliferation and apoptosis. In grade IV GBM, TF genes include tumor suppressor genes (ERCC2, KDM5C,

Table 3. Pathways implicated in astrocytoma grade II - IV.

\begin{tabular}{|c|c|c|c|c|c|c|}
\hline Function & Pathways & 离 & 豊 & $\sum_{\substack{0 \\
\text { D }}}$ & $\sum_{\substack{0 \\
\text { D }}}$ & 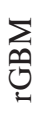 \\
\hline \multirow[t]{6}{*}{ Evasion of Apoptosis } & APOPTOSIS & & & $\mathrm{Y}$ & $\mathrm{Y}$ & \\
\hline & CLASS I MHC PATHWAY & $\mathrm{Y}$ & $\mathrm{Y}$ & $\mathrm{Y}$ & Y & $\mathrm{Y}$ \\
\hline & NATURAL KILLER CELL MEDIATED CYTOTOXICITY & & & $\mathrm{Y}$ & & \\
\hline & P53 SIGNALING PATHWAY & $\mathrm{Y}$ & & $\mathrm{Y}$ & & \\
\hline & P73PATHWAY & $\mathrm{Y}$ & $\mathrm{Y}$ & $\mathrm{Y}$ & Y & \\
\hline & TAP63 PATHWAY (LKB1) & $\mathrm{Y}$ & & & $\mathrm{Y}$ & \\
\hline \multirow[t]{3}{*}{ Growth and survival } & NEUROTROPHIN SIGNALING PATHWAY & & $\mathrm{Y}$ & $\mathrm{Y}$ & & \\
\hline & PHOSPHATIDYLINOSITOL SIGNALING SYSTEM & $\mathrm{Y}$ & $\mathrm{Y}$ & $\mathrm{Y}$ & & \\
\hline & PI3KCI AKT PATHWAY & & & $\mathrm{Y}$ & & \\
\hline \multirow[t]{2}{*}{ Homeostasis } & TCR PATHWAY & $\mathrm{Y}$ & & $\mathrm{Y}$ & & \\
\hline & TOLL RECEPTOR CASCADES & & & $\mathrm{Y}$ & & \\
\hline \multirow[t]{4}{*}{ Metastasis } & RAC1 PATHWAY & & & & $\mathrm{Y}$ & \\
\hline & RHO REG PATHWAY & $\mathrm{Y}$ & & $\mathrm{Y}$ & & \\
\hline & SEMAPHORIN INTERACTIONS & $\mathrm{Y}$ & & $\mathrm{Y}$ & $\mathrm{Y}$ & $\mathrm{Y}$ \\
\hline & UPA UPAR PATHWAY & & & $\mathrm{Y}$ & & \\
\hline \multirow[t]{4}{*}{ Proliferation } & MTOR PATHWAY & $\mathrm{Y}$ & $\mathrm{Y}$ & $\mathrm{Y}$ & & \\
\hline & MYC ACTIV PATHWAY & $\mathrm{Y}$ & & & & \\
\hline & NOTCH SIGNALING PATHWAY & & & $\mathrm{Y}$ & & \\
\hline & TXA2 PATHWAY & $\mathrm{Y}$ & & $\mathrm{Y}$ & & \\
\hline \multirow{23}{*}{$\begin{array}{l}\text { Proliferation and } \\
\text { invasion }\end{array}$} & CELL ADHESION MOLECULES CAMS & $\mathrm{Y}$ & $\mathrm{Y}$ & $\mathrm{Y}$ & $\mathrm{Y}$ & $\mathrm{Y}$ \\
\hline & CHEMOKINE SIGNALING PATHWAY & $\mathrm{Y}$ & $\mathrm{Y}$ & $\mathrm{Y}$ & & $\mathrm{Y}$ \\
\hline & COLLAGENS & $\mathrm{Y}$ & $\mathrm{Y}$ & $\mathrm{Y}$ & $\mathrm{Y}$ & $\mathrm{Y}$ \\
\hline & CYTOKINE -CYTOKINE RECEPTOR INTERACTION & $\mathrm{Y}$ & $\mathrm{Y}$ & $\mathrm{Y}$ & & \\
\hline & ECM PATHWAY & & $\mathrm{Y}$ & $\mathrm{Y}$ & Y & $\mathrm{Y}$ \\
\hline & ERBB SIGNALING PATHWAY & $\mathrm{Y}$ & $\mathrm{Y}$ & $\mathrm{Y}$ & & \\
\hline & ERK1 ERK2 MAPK PATHWAY & & & $\mathrm{Y}$ & & \\
\hline & FOCAL ADHESION & $\mathrm{Y}$ & $\mathrm{Y}$ & $\mathrm{Y}$ & Y & $\mathrm{Y}$ \\
\hline & GAP JUNCTION & $\mathrm{Y}$ & $\mathrm{Y}$ & & Y & $\mathrm{Y}$ \\
\hline & INTEGRIN PATHWAY & $\mathrm{Y}$ & $\mathrm{Y}$ & $\mathrm{Y}$ & Y & $\mathrm{Y}$ \\
\hline & LYSOPHOSPHOLID PATHWAY & $\mathrm{Y}$ & $\mathrm{Y}$ & & & Y \\
\hline & MAPK SIGNALING PATHWAY & & & $\mathrm{Y}$ & & \\
\hline & MATRISOME ASSOCIATED & $\mathrm{Y}$ & $\mathrm{Y}$ & $\mathrm{Y}$ & Y & $\mathrm{Y}$ \\
\hline & PDGF PATHWAY & & & $\mathrm{Y}$ & & \\
\hline & PLATELET ACTIVATION SIGNALING & $\mathrm{Y}$ & $\mathrm{Y}$ & $\mathrm{Y}$ & $\mathrm{Y}$ & $\mathrm{Y}$ \\
\hline & REGULATION OF ACTIN CYTOSKELETON & $\mathrm{Y}$ & $\mathrm{Y}$ & $\mathrm{Y}$ & $\mathrm{Y}$ & $\mathrm{Y}$ \\
\hline & REGULATION OF HYPOXIA & $\mathrm{Y}$ & $\mathrm{Y}$ & & Y & $\mathrm{Y}$ \\
\hline & SIGNALING BY EGFR IN CANCER & $\mathrm{Y}$ & $\mathrm{Y}$ & $\mathrm{Y}$ & $\mathrm{Y}$ & $\mathrm{Y}$ \\
\hline & SIGNALING BY FGFR & & & $\mathrm{Y}$ & & \\
\hline & SYNDECAN PATHWAY & & & $\mathrm{Y}$ & & \\
\hline & VEGF SIGNALING PATHWAY & $\mathrm{Y}$ & & $\mathrm{Y}$ & & \\
\hline & WNT BETA CATENIN PATHWAY & & $\mathrm{Y}$ & & $\mathrm{Y}$ & $\mathrm{Y}$ \\
\hline & WNT SIGNALING PATHWAY & & $\mathrm{Y}$ & $\mathrm{Y}$ & $\mathrm{Y}$ & $\mathrm{Y}$ \\
\hline \multirow[t]{3}{*}{ Tumorigenesis } & GLIOMA & $\mathrm{Y}$ & & & & \\
\hline & GNRH SIGNALING PATHWAY & $\mathrm{Y}$ & $\mathrm{Y}$ & & & \\
\hline & HDAC CLASSI PATHWAY & $\mathrm{Y}$ & & & & \\
\hline
\end{tabular}

*This is a summary from 100 most significantly enriched pathways in AS II-IV $(P \leq 0.0013$ and FDR $\leq 0.0018)$. Y- indicates significant enrichment of that pathway. 
SMARCA4: upregulated) and oncogenes (BCL3, ETV1/4, JUN, NFKB2, MYC: upregulated; FOXP1, MLLT3, NF1, REL: down-regulated).

\section{Key glioma pathway genes that were differentially expressed in pGBM}

Hallmark pathways in cancer such as evasion of apoptosis (e.g., Class I MHC pathway), growth and survival (e.g., Phosphatidylinositol signaling pathway), and proliferation and invasion (e.g., EGFR signaling, collagens, ECM, CAM, Chemokines, ERBB signaling) were enriched in grades II-IV subtypes in our study (Table 3). Differentially expressed genes from all grades of astrocytoma were significantly enriched in pathways involved in cell proliferation and tumor invasion along with pathways involved in normal brain function $(P=0.0013$ and FDR $=$ 0.0018 ). Key genes in glioma pathways (de novo/secondary pathway), such as cytokine-cytokine receptor interaction genes EGF, TGF $\alpha$, PDGF and their receptors EGFR (oncogene) and PDGFR were significantly over-expressed in pGBM. ErbB signaling pathway genes SHC (SHC (Src Homology 2 Domain Containing) Transforming Protein 1) were significantly over-expressed in pGBM whereas SOS2 (SOS Ras/Rho Guanine Nucleotide Exchange Factor 2) gene was downregulated in pGBM. Calcium signaling pathway genes PLC $\gamma$ (phospholipase $\mathrm{C}$ gamma) were significantly over-expressed in pGBM and
CAMK2G (Calcium/Calmodulin Dependent Protein Kinase II Gamma) gene was downregulated. Genes involved in The MAPK/ERK pathway (also known as the Ras-Raf-MEK-ERK pathway) such as oncogenes Ras, Raf, MEK (MAPK/ERK Kinase), ERK, and AKT1/2 genes were upregulated in pGBM. MTOR (Mechanistic Target Of Rapamycin) signaling pathway genes such as PI3K (Phosphatidylinositol-4, 5-Bisphosphate 3-Kinase) genes, $\mathrm{PKB} /+\mathrm{AKT}$, and MTOR genes were significantly upregulated in pGBM. Genes in p53 signaling pathway, such as TP14/TP16/ARF/CDKN2A (Cyclin-Dependent Kinase Inhibitor 2A), CCND1 (cyclinD1), CDK4/6, and E2F transcription factors 1-6 were significantly upregulated in pGBM and TP53 (tumor protein 53) gene was significantly downregulated in pGBM. Tumor suppressor gene PTEN (Phosphatase and Tensin Homolog) gene was significantly downregulated in all astrocytoma grades (Fig. 4).

\section{DISCUSSION}

Our study was focused on describing similarities and differences in gene expression in adult astrocytoma samples by transcriptome analysis. Our investigation revealed a distinct gene expression pattern between grade II and astrocytoma grade IV. This observation was similar to

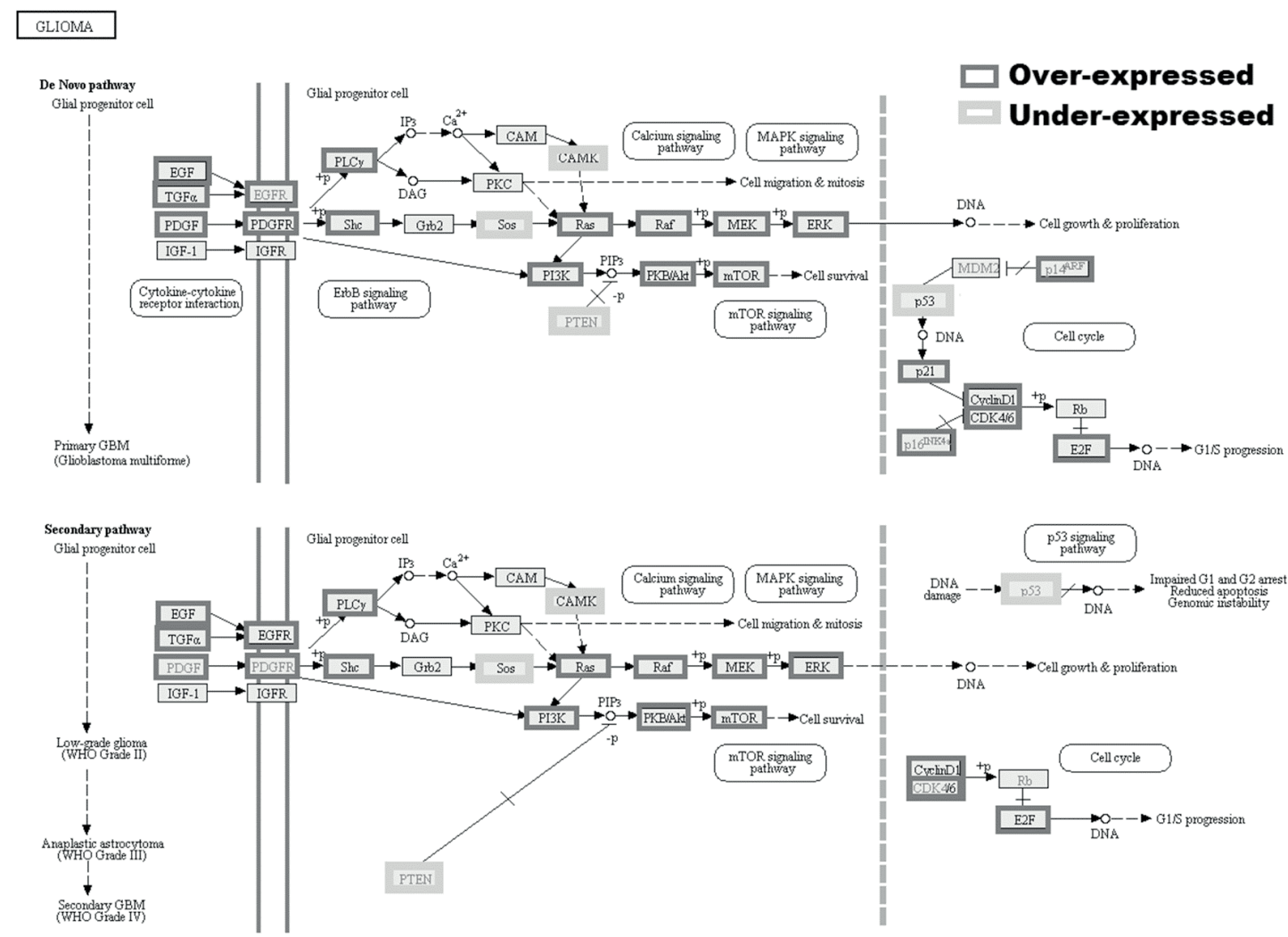

Fig. 4. Gene expression changes in glioma pathway. Key KEGG glioma pathway genes in our study. In this figure we have highlighted key KEGG glioma pathway genes (hsa: 04060) that were observed in pGBM. Upregulated genes (in red colored rectangles) and downregulated genes (in blue colored rectangles) from our study were highlighted in this figure. Genes that were not highlighted by colored rectangles were either absent or unchanged in our study. 
previous studies which described that AS-II grade is less proliferative and less aggressive compared to grade IV astrocytoma ${ }^{4}$. IL2-STAT5 pathway genes were significantly enriched in grade IV astrocytoma alone and previous studies have reported that STAT5 regulates glioma cell invasion by pathways dependent and independent of STAT5 DNA binding ${ }^{45}$. The number of differentially expressed genes per sample in pGBM $(n=58)$ and AS -II $(n=34)$ were higher than those of AS-III ( $n=13)$, sGBM $(n=19)$, and rGBM $(n=19)$, however this difference could partly due to higher number of samples in these two subtypes.

Our data has confirmed gene expression pattern for genes that were previously reported to characterize the GBM IV, with an exception of expression pattern in genes MDM2, MDM4, AXL, FGFR3, and MERTK, which were reported to be over-expressed in glioma and were significantly under-expressed in all GBM subtypes in our study (pGBM (-2.92 log FC), sGBM (-2.14 log FC), and rGBM $(-2.57 \log$ FC). MDM2 and MDM4 are known to inhibit a p53-dependent growth control ${ }^{46-48}$. However, amplification of the CDK4 gene and over-expression of cyclin D1 are suggested to have similar effects to $\mathrm{p} 16$ or $\mathrm{pRb}$ inactivation and these mechanisms may provide additional alternatives to subvert cell-cycle control and facilitate progression to GBM (ref. ${ }^{49}$ ). CDK4, CCND1 (CyclinD1) and $\mathrm{E} 2 \mathrm{~F}$ were over-expressed in primary GBM samples in our study, which may have contributed to the evasion of cell cycle control and facilitate G1/S progression (Fig. 4).

BIRC3 (CIAP2) encodes a member of the IAP family of proteins that inhibit apoptosis by binding to tumor necrosis factor receptor-associated factors TRAF1 and TRAF2. This gene was reported to be a transcriptional target of NF-kB/p65 and reduced levels of p65 renders glioma cells more sensitive to the cytotoxic effects of TNF- $\alpha$, suggesting a good therapeutic target for the treatment of malignant gliomas ${ }^{50-51}$.

NTRK3 was reported to be under-expressed in highgrade gliomas due to DNA methylation changes, and was also significantly under-expressed in our study (pGBM (-2.09 log FC), sGBM (-1.22 log FC), and rGBM (-1.71 $\log$ FC) $\left(\right.$ ref. $\left.^{52}\right)$. TGFBI (transforming growth factor beta induced) gene was significantly over-expressed in our primary GBM samples. It encodes an RGD-containing protein that binds to type I, II and IV collagens. Collagens were also significantly over-expressed in our pGBM samples. In our study, pGBM samples were enriched in the genes involved in TGFBI module, namely, TGFBI, Collagens, MMP2 (matrix metalloproteinase 2), SPARC (secreted protein, acidic and rich in cysteine), and fibronectin (FN). TGFBI module may present as an alternative signaling pathway (to the canonical SMAD-mediated) TGF-beta signaling pathway in GBM ( ref. $\left.^{53}\right)$. All the genes that are associated with this alternative pathway were significantly over-expressed in our AS grade IV pGBM samples (see supplementary Table 2 (S2)).

Genes exclusively over-expressed in AS II include the transcription factors such as THRA (Thyroid Hormone Receptor) gene which is a nuclear hormone receptor that can act as a repressor or activator of transcription and play a role in development (NOTCH1-mediated pathway for NF-KB activity modulation), KLF15 (which is a negative regulator of TP53 acetylation and also inhibits NF-kappa-B activation through repression of EP300dependent RELA acetylation), and FHL1 genes, Protein kinase gene, DCLK2, and Cytokine and growth factors, IL17D, CMTM4 and SEMA6A (ref. ${ }^{54}$ ).

Interestingly, IL-17D gene expression was reported to be suppressed in more advanced, higher grade gliomas (grade III and grade IV) relative to less advanced, lower stage gliomas (grade II oligodendroglioma). IL-17D expression was also suppressed in Grade IV GBM when compared to Grade III astrocytoma, high expression of IL-17D in tumor biopsies correlated with a greater survival time for a subset of patients with grade IV GBM $\left(\right.$ ref. $\left.^{55}\right)$. Oncogenes TFRC, MDM2, ETV5, and Transcription factor ENO1, and cytokine and growth factor PTN are exclusively under-expressed in the AS II subtype in our study. Interestingly, MDM2 gene which was reported to be over-expressed in gliomas has under or un-altered expression in our study ${ }^{56}$. TFRC was postulated to be expressed in the normal brain and may be dysregulated in brain cancers ${ }^{57}$. Previous studies suggested ENO1 overexpression is associated with tumor progression in highgrade gliomas ${ }^{58}$. ENO1 was exclusively under-expressed in astrocytoma subtype AS II and non-significant $(P<$ 0.05 ) over-expression of ENO1 gene was observed in ASIII and grade IV in our study. ITM2C (BRI3) was the only gene that was exclusively over-expressed in AS III subtype. ITM2C is a negative regulator of beta-amyloid peptide production, which inhibits the processing of APP by blocking its access to alpha- and beta-secretase. ITM2C was suggested to play a role in TNF-induced cell death and neuronal differentiation ${ }^{59}$. SOX4 and SALL3 genes were exclusively over-expressed in secondary GBM in our study. SOX4 (sex determining region Y-box 4) was reported to be over-expressed in GBM (ref. $\left.{ }^{53}\right)$. SOX4 was shown to transcriptionally activate EGFR by binding to its promoter ${ }^{60}$. Other growth factors that are reported to be targets of SOX4 are FGFRL1 and IGF2R (see supplementary Table 4 (S4).

Gene set enrichment analysis of differentially expressed genes in each AS subtype revealed important cancer-relevant signaling processes. Transcription factors/co-factors in astrocytoma grades were enriched in cell cycle control, DNA repair, TGF BETA signaling, hypoxia, proliferation and apoptosis, with the exception of grade III where enrichment of cell cycle (E2F) targets and chromatin binding was observed. Pathway analysis of each astrocytoma subtype revealed a significant enrichment of hallmark pathways in cancer such as evasion of apoptosis (e.g., Class I MHC pathway), growth and survival (e.g., Phosphatidylinositol signaling pathway), and proliferation and invasion (e.g., EGFR signaling, collagens, ECM, CAM, Chemokines, ERBB signaling) (see Table 3). Mapping of the genes that were differentially expressed genes in AS subtypes on KEGG glioma pathway revealed key genes involved in glioma-genesis (Fig. 4). 


\section{CONCLUSION}

In conclusion, this study revealed similarities and differences in gene expression levels between grades II-IV AS subtypes by comparing their gene expression levels to those of normal brain samples, especially between grade II and grade IV astrocytoma. We identified genes that were exclusively expressed in each of the AS subtype. Pathway analysis of differentially expressed genes in each AS subtype revealed important cancer-relevant signaling processes. Furthermore, we may have identified an alternative signaling pathway (to the canonical SMAD-mediated) TGF-beta signaling pathway in GBM. A literature review of the genes identified in this study revealed that many were implicated in glioma pathogenesis and other cancers. We confirmed existing findings and shed light on some important genes and molecular processes that will improve our understanding of glioma biology. However, an integrated study involving much larger cohort augmented with methylation, whole genome and gene expression data from both tumor and normal brain samples will provide a greater understanding of glioma development and aid in personalized therapy. We believe our findings will provide new insights into the mechanisms of glioma pathogenesis and provide a valuable resource for future studies.

\section{ABBREVIATIONS}

WHO: World Health Organization; AS: Astrocytoma; GBM: Glioblastoma ( $\mathrm{p}$ - Primary, S - Secondary, r Recurrent); FC: Fold change; TF: Transcription factor/ co-factor; hsa: Homo sapiens.

Acknowledgement: This work was supported by grants NPU I LO1304 and RVO: 61989592 from the Czech Ministry of Education and LF_2016_013.

Author contributions: All authors had full access to all the data in the study and take responsibility for the integrity of the data and the accuracy of the data analysis; NN, PR: study concept and design, acquisition of data; PR: Data analysis and interpretation; PR, NN, JE: manuscript drafting; NN, PR, JR, ZK: critical revision; PR: statistical analysis.

Conflict of interest statement: The authors state that there are no conflicts of interest regarding the publication of this article.

\section{REFERENCES}

1. Ohgaki H, Kleihues P. Population-based studies on incidence, survival rates, and genetic alterations in astrocytic and oligodendroglial gliomas. J Neuropathol Exp Neurol 2005; 64:479-89.

2. Canoll P, Goldman JE. The interface between glial progenitors and gliomas. Acta Neuropathologica 2008;116:465-77.

3. Chen J, McKay Renée M, Parada Luis F. Malignant Glioma: Lessons from Genomics, Mouse Models, and Stem Cells. Cell 2012;149:36-47.

4. Louis DN, Ohgaki H, Wiestler OD, Cavenee WK, Burger PC, Jouvet A, Scheithauer BW, Kleihues P. The 2007 WHO classification of tumours of the central nervous system. Acta Neuropathol 2007;114:97-109.
5. Tonn JC WM, Rutka JT, Grossman SA. Neuro-Oncology of CNS Tumors. Springer Science + Business Media; 2006.

6. Kelly P. Gliomas. Survival, origin and early detection. Surgical Neurology International 2010; 1:96.

7. Johnson BE, Mazor T, Hong C, Barnes M, Aihara K, McLean CY, Fouse SD, Yamamoto S, Ueda H, Tatsuno K. Mutational Analysis Reveals the Origin and Therapy-Driven Evolution of Recurrent Glioma. Science 2013;343:189-93.

8. Ohgaki $\mathrm{H}$, Kleihues P. Genetic alterations and signaling pathways in the evolution of gliomas. Cancer Science 2009;100:2235-41.

9. Lind-Landström T, Habberstad AH, Torp SH. Proliferative activity and histopathological features in diffuse grade II astrocytomas. APMIS 2012,120:640-7.

10. Smoll NR, Hamilton B. Incidence and relative survival of anaplastic astrocytomas. Neuro-Oncology 2014;16:1400-7.

11. Nuño M, Birch K, Mukherjee D, Sarmiento JM, Black KL, Patil CG. Survival and Prognostic Factors of Anaplastic Gliomas. Neurosurgery 2013;73:458-65.

12. Ohgaki $\mathrm{H}$, Kleihues P. The Definition of Primary and Secondary Glioblastoma. Clinical Cancer Research 2012;19:764-72.

13. Sturm D, Bender S, Jones DTW, Lichter P, Grill J, Becher O, Hawkins $C$ Majewski J, Jones C, Costello JF, lavarone A, Aldape K, Brennan CW, Jabado N, Pfister SM. Paediatric and adult glioblastoma: multiform (epi)genomic culprits emerge. Nature Reviews Cancer 2014;14:92-107.

14. Parsons DW, Jones $S$, Zhang $X$, Lin JCH, Leary RJ, Angenendt $P$ Mankoo P, Carter H, Siu IM, Gallia GL, Olivi A, McLendon R, Rasheed BA, Keir S, Nikolskaya T, Nikolsky Y, Busam DA, Tekleab H, Diaz LA Jr, Hartigan J, Smith DR, Strausberg RL, Marie SK, Shinjo SM, Yan $\mathrm{H}$, Riggins GJ, Bigner DD, Karchin R, Papadopoulos N, Parmigiani G, Vogelstein B, Velculescu VE, Kinzler KW. An Integrated Genomic Analysis of Human Glioblastoma Multiforme. Science 2008; 321:1807-12.

15. Brennan Cameron W, Verhaak Roel GW, McKenna A, Campos B, Noushmehr H, Salama Sofie R, Zheng S, Chakravarty D, Sanborn JZ, Berman SH, Beroukhim R, Bernard B, Wu CJ, Genovese G, Shmulevich I, Barnholtz-Sloan J, Zou L, Vegesna R, Shukla SA, Ciriello G, Yung WK, Zhang W, Sougnez C, Mikkelsen T, Aldape K, Bigner DD, Van Meir EG, Prados M, Sloan A, Black KL, Eschbacher J, Finocchiaro G, Friedman W, Andrews DW, Guha A, lacocca M, O'Neill BP, Foltz G, Myers J, Weisenberger DJ, Penny R, Kucherlapati R, Perou CM, Hayes DN, Gibbs R, Marra M, Mills GB, Lander E, Spellman P, Wilson R, Sander C, Weinstein J, Meyerson M, Gabriel S, Laird PW, Haussler D, Getz G, Chin L; TCGA Research Network. The Somatic Genomic Landscape of Glioblastoma. Cell 2013;155:462-77.

16. Jones DTW, Gronych J, Lichter P, Witt O, Pfister SM. MAPK pathway activation in pilocytic astrocytoma. Cellular and Molecular Life Sciences 2011;69:1799-811.

17. Cancer Genome Atlas Research N. Comprehensive genomic characterization defines human glioblastoma genes and core pathways. Nature 2008;455:1061-8.

18. Cooper LAD, Gutman DA, Long Q, Johnson BA, Cholleti SR, Kurc T, Saltz JH, Brat DJ, Moreno CS. The Proneural Molecular Signature Is Enriched in Oligodendrogliomas and Predicts Improved Survival among Diffuse Gliomas. PLoS ONE 2010;5:e12548.

19. Noushmehr H, Weisenberger DJ, Diefes K, Phillips HS, Pujara K, Berman BP, Pan F, Pelloski CE, Sulman EP, Bhat KP, Verhaak RG, Hoadley KA, Hayes DN, Perou CM, Schmidt HK, Ding L, Wilson RK, Van Den Berg D, Shen H, Bengtsson H, Neuvial P, Cope LM, Buckley J, Herman JG, Baylin SB, Laird PW, Aldape K; Cancer Genome Atlas Research Network. Identification of a CpG Island Methylator Phenotype that Defines a Distinct Subgroup of Glioma. Cancer Cell 2010;17:510-22.

20. Verhaak RGW, Hoadley KA, Purdom E, Wang V, Qi Y, Wilkerson MD, Miller CR, Ding L, Golub T, Mesirov JP, Alexe G, Lawrence M, O'Kelly M, Tamayo P, Weir BA, Gabriel S, Winckler W, Gupta S, Jakkula L, Feiler HS, Hodgson JG, James CD, Sarkaria JN, Brennan C, Kahn A, Spellman PT, Wilson RK, Speed TP, Gray JW, Meyerson M, Getz G, Perou CM, Hayes DN; Cancer Genome Atlas Research Network. Integrated Genomic Analysis Identifies Clinically Relevant Subtypes of Glioblastoma Characterized by Abnormalities in PDGFRA, IDH1, EGFR, and NF1. Cancer Cell 2010;17:98-110.

21. Carro MS, Lim WK, Alvarez MJ, Bollo RJ, Zhao X, Snyder EY, Sulman EP, Anne SL, Doetsch F, Colman H, Lasorella A, Aldape K, Califano A, lavarone $A$. The transcriptional network for mesenchymal transformation of brain tumours. Nature 2009;463:318-25. 
22. Deshmukh H, Yu J, Shaik J, MacDonald TJ, Perry A, Payton JE, Gutmann DH, Watson MA, Nagarajan R. Identification of transcriptional regulatory networks specific to pilocytic astrocytoma. BMC Med Genomics 2011;4:57. doi:10.1186/1755-8794-4-57

23. Jornsten $R$, Abenius T, Kling T, Schmidt $L$, Johansson E, Nordling TE, Nordlander B, Sander C, Gennemark P, Funa K, Nilsson B, Lindahl L, Nelander S. Network modeling of the transcriptional effects of copy number aberrations in glioblastoma. Mol Syst Biol 2011;7:486.

24. Setty M, Helmy K, Khan AA, Silber J, Arvey A, Neezen F, Agius P, Huse JT, Holland EC, Leslie CS. Inferring transcriptional and microRNAmediated regulatory programs in glioblastoma. Molecular Systems Biology 2012;8:605. doi:10.1038/msb.2012.37

25. Wang C, Funk CC, Eddy JA, Price ND. Transcriptional Analysis of Aggressiveness and Heterogeneity across Grades of Astrocytomas. PLoS ONE 2013;8:e76694.

26. Hunter S, Young A, Olson J, Brat DJ, Bowers G, Wilcox JN, Jaye D, Mendrinos S, Neish A. Differential Expression between Pilocytic and Anaplastic Astrocytomas: Identification of Apolipoprotein D as a Marker for Low-Grade, Non-Infiltrating Primary CNS Neoplasms. J Neuropathol Exp Neurol 2002;61:275-81.

27. Rickman DS, Bobek MP, Misek DE, Kuick R, Blaivas M, Kurnit DM, Taylor J, Hanash SM. Distinctive molecular profiles of high-grade and low-grade gliomas based on oligonucleotide microarray analysis. Cancer Res 2001;61:6885-91.

28. Seifert M, Garbe M, Friedrich B, Mittelbronn M, Klink B. Comparative transcriptome reveals similarities and differences between astrocytoma grades. BMC Cancer 2015;15:952. doi:10.1186/s12885-0151939-9

29. Liao Y, Smyth GK, Shi W: The Subread aligner. fast, accurate and scalable read mapping by seed-and-vote. Nucleic Acids Research 2013;41:e108-e108.

30. McCarthy DJ, Chen Y, Smyth GK. Differential expression analysis of multifactor RNA-Seq experiments with respect to biological variation. Nucleic Acids Research 2012;40:4288-97.

31. Futreal PA, Coin L, Marshall M, Down T, Hubbard T, Wooster R, Rahman N, Stratton MR. A census of human cancer genes. Nature Reviews Cancer 2004;4:177-83.

32. Zhao S, Guo Y, Sheng Q, Shyr Y. Advanced Heat Map and Clustering Analysis Using Heatmap3. BioMed Research International 2014;2014:1-6

33. Bai J, Mei P-J, Liu H, Li C, Li W, Wu Y-P, Yu Z-Q, Zheng J-N. BRG1 expression is increased in human glioma and controls glioma cell proliferation, migration and invasion in vitro. Journal of Cancer Research and Clinical Oncology 2012;138:991-8.

34. Han Y, Wu Z, Wu T, Huang Y, Cheng Z, Li X, Sun T, Xie X, Zhou Y, Du $Z$. Tumor-suppressive function of long noncoding RNA MALAT1 in glioma cells by downregulation of MMP2 and inactivation of ERK/ MAPK signaling. Cell Death Dis 2016;7:e2123.

35. Artesi M, Kroonen J, Bredel M, Nguyen-Khac M, Deprez M Schoysman L, Poulet C, Chakravarti A, Kim H, Scholtens D, Seute T, Rogister B, Bours V, Robe PA. Connexin 30 expression inhibits growth of human malignant gliomas but protects them against radiation therapy. Neuro-Oncology 2014.

36. Lambiv WL, Vassallo I, Delorenzi M, Shay T, Diserens AC, Misra A Feuerstein B, Murat A, Migliavacca E, Hamou MF, Sciuscio D, Burger R, Domany E, Stupp R, Hegi ME. The Wnt inhibitory factor 1 (WIF1) is targeted in glioblastoma and has a tumor suppressing function potentially by induction of senescence. Neuro-Oncology 2011;13:73647.

37. Tong $H, Y u X, L u X$, Wang P. Downregulation of solute carriers of glutamate in gliosomes and synaptosomes may explain local brain metastasis in anaplastic glioblastoma. IUBMB Life 2015;67:306-11.

38. Liu S, Yin F, Fan W, Wang S, Guo X-r, Zhang J-n, Tian Z-m, Fan M Over-expression of BMPR-IB reduces the malignancy of glioblastoma cells by upregulation of p21 and p27Kip1. J Exp Clin Cancer Res 2012,31:52.

39. Jensen SS, Aaberg-Jessen C, Christensen KG, Kristensen B. Expression of the lysosomal-associated membrane protein-1 (LAMP-1) in astrocytomas. Int J Clin Exp Pathol 2013;6:1294-5.

40. Fan Y, Potdar AA, Gong Y, Eswarappa SM, Donnola S, Lathia JD, Hambardzumyan D, Rich JN, Fox PL. Profilin-1 phosphorylation directs angiocrine expression and glioblastoma progression through HIF-1a accumulation. Nature Cell Biology 2014;16:445-56.

41. Takahashi M, Shimajiri S, Izumi H, Hirano G, Kashiwagi E, Yasuniwa Y, Wu Y, Han B, Akiyama M, Nishizawa S, Sasaguri Y, Kohno K. Y-box binding protein-1 is a novel molecular target for tumor vessels. Cancer Science 2010;101:1367-73.

42. Miotto E. Frequent Aberrant Methylation of the $\mathrm{CDH} 4 \mathrm{Gene}$ Promoter in Human Colorectal and Gastric Cancer. Cancer Research 2004;64:8156-9.

43. Phillips HS, Kharbanda S, Chen R, Forrest WF, Soriano RH, Wu TD, Misra A, Nigro JM, Colman H, Soroceanu L, Williams PM, Modrusan Z, Feuerstein BG, Aldape K. Molecular subclasses of high-grade glioma predict prognosis, delineate a pattern of disease progression, and resemble stages in neurogenesis. Cancer Cell 2006;9:157-73.

44. Xie Q, Flavahan William A, Bao S, Rich J. The Tailless Root of Glioma: Cancer Stem Cells. Cell Stem Cell 2014;15:114-6.

45. Cao S, Wang C, Zheng Q, Qiao Y, Xu K, Jiang T, Wu A. STAT5 regulates glioma cell invasion by pathways dependent and independent of STAT5 DNA binding. Neurosci Lett 2011;487:228-33.

46. Pedeutour-Braccini Z, Burel-Vandenbos F, Gozé C, Roger C, Bazin A, Costes-Martineau V, Duffau H, Rigau V. Microfoci of malignant progression in diffuse low-grade gliomas: towards the creation of an intermediate grade in glioma classification? Virchows Archiv 2015;466:433-44.

47. Riemenschneider MJ, Buschges R, Wolter M, Reifenberger J, Bostrom J, Kraus JA, Schlegel U, Reifenberger G. Amplification and overexpression of the MDM4 (MDMX) gene from 1q32 in a subset of malignant gliomas without TP53 mutation or MDM2 amplification. Cancer Res 1999;59:6091-6.

48. Riemenschneider MJ, Knobbe CB, Reifenberger G. Refined mapping of $1 \mathrm{q} 32$ amplicons in malignant gliomas confirmsMDM4 as the main amplification target. International Journal of Cancer 2003;104:752-7.

49. Schmidt EE, Ichimura K, Reifenberger G, Collins VP. CDKN2 (p16/ MTS1) gene deletion or CDK4 amplification occurs in the majority of glioblastomas. Cancer Res 1994;54:6321-4

50. Gressot LV, Doucette T, Yang Y, Fuller GN, Manyam G, Rao A, Latha K, Rao G. Analysis of the inhibitors of apoptosis identifies BIRC3 as a facilitator of malignant progression in glioma. Oncotarget $2016 \mathrm{Apr}$ 8. doi: 10.18632/oncotarget.8657. [Epub ahead of print]

51. Zhao X, Laver T, Hong SW, Twitty GB, DeVos A, DeVos M, Benveniste EN, Nozell SE. An NF-kB p65-clAP2 link is necessary for mediating resistance to TNF- $a$ induced cell death in gliomas. Journal of NeuroOncology 2011;102:367-81.

52. Palani M, Arunkumar R, Vanisree AJ. Methylation and Expression Patterns of Tropomyosin-Related Kinase Genes in Different Grades of Glioma. Neuromol Med 2014;16:529-39.

53. Lin B, Madan A, Yoon J-G, Fang X, Yan X, Kim T-K, Hwang D, Hood L, Foltz G. Massively Parallel Signature Sequencing and Bioinformatics Analysis Identifies Up-Regulation of TGFBI and SOX4 in Human Glioblastoma. PLoS ONE 2010; 5:e10210.

54. Wolter M, Werner T, Malzkorn B, Reifenberger G. Role of microRNAs Located on Chromosome Arm 10q in Malignant Gliomas. Brain Pathology 2015;26:344-58.

55. O'Sullivan T, Saddawi-Konefka R, Gross E, Tran M, Mayfield Stephen P Ikeda H, Bui Jack D. Interleukin-17D Mediates Tumor Rejection through Recruitment of Natural Killer Cells. Cell Reports 2014;7:98998.

56. Soni D, King JAJ, Kaye AH, Hovens CM: Genetics of glioblastoma multiforme. mitogenic signaling and cell cycle pathways converge. Journal of Clinical Neuroscience 2005;12:1-5.

57. Hänninen MM, Haapasalo J, Haapasalo H, Fleming RE, Britton RS, Bacon BR, Parkkila S. Expression of iron-related genes in human brain and brain tumors. BMC Neuroscience 2009;10:36.

58. Song Y, Luo Q, Long H, Hu Z, Que T, Zhang Xa, Li Z, Wang G, Yi L, Liu Z Fang W, Qi S. Alpha-enolase as a potential cancer prognostic marker promotes cell growth, migration, and invasion in glioma. Molecular Cancer 2014;13:65

59. Matsuda S, Matsuda Y, D'Adamio L. BRI3 Inhibits Amyloid Precursor Protein Processing in a Mechanistically Distinct Manner from Its Homologue Dementia Gene BRI2. Journal of Biological Chemistry 2009;284:15815-25.

60. Scharer CD, McCabe CD, Ali-Seyed M, Berger MF, Bulyk ML, Moreno CS. Genome-Wide Promoter Analysis of the SOX4 Transcriptional Network in Prostate Cancer Cells. Cancer Research 2009;69:709-17.

\section{Supplemental Material: \\ The online version of this article (doi: $10.5507 /$ bp.2017.020) offers supplemental material.}

